NYU-TH-07/12/24

\title{
Electrodynamic Metanuclei
}

\author{
Gregory Gabadadze and Rachel A. Rosen \\ Center for Cosmology and Particle Physics \\ Department of Physics, New York University, New York, NY, 10003, USA
}

\begin{abstract}
A relativistic system of electrically charged fermions and oppositely charged massive scalars with no self-interactions, is argued to have a long-lived collective state with a net charge. The charge is residing near the surface of the spherically-symmetric state, while the interior consists of the condensed scalars, that are neutralized by the fermions. The metastability is achieved by competition of the negative pressure of the scalar condensate, against the positive pressure, mainly due to the fermions. We consider such metanuclei made of helium-4 nuclei and electrons, below nuclear but above atomic densities. Typical metanuclei represent charged balls of the atomic size, colossal mass, electric charge and excess energy. Unlike an ordinary nucleus, the charge of a metanucleus scales proportionately to its radius. The quantum mechanical decay through tunneling, and vacuum instability via pair-creation, are both suppressed for large values of the electric charge. Similar states could also be composed of other charged (pseudo)scalars, such as the pions, scalar supersymmetric partners, or in general, spin-0 states of new physics.
\end{abstract}




\section{Introduction and summary}

The purpose of this work is to show that in a relativistic system of $N+Q$ fermions, each of charge $g$, and $N$ oppositely charged massive scalars, with no non-linear self-interactions, there may exist a metastable long-lived spherically symmetric ball with the following identity: The excess charge $g Q$ is residing on a surface of the ball, while in its neutral interior there are $N$ condensed scalars, that act collectively as a macroscopic state of a large occupation number, and also the $N$ fermions playing the role of spectators that neutralize the bulk scalar charg 1 . The radius of the ball $R$ scales linearly with the charge $g Q$, and the electric field near its surface, $g Q /\left(4 \pi R^{2}\right)$, decreases with increasing charge, in the regime of applicability of our arguments, $N^{1 / 3} \ll g^{2} Q \ll g N^{2 / 3}$.

The physical reason for (meta)stability of such a ball is that the scalar condensate gives an attractive negative pressure which balances against the repulsive pressure, mainly due to the positive energy of the fermions. As a result, the energy functional has a minimum around the point of balance. The total energy stored in the ball is greater than the energy of $N$ neutral scalar-fermion atoms plus free $Q$ fermions. Hence, the minimum of the energy functional is only a local one - the condensate ball can decay into the atomic state by tunneling. However, for large values of the charge, both the tunneling and the vacuum instability through quantum pair-creation, are suppressed.

The spectrum of small perturbations above the scalar condensate in the bulk of the ball has a mass gap that equals to $2 m_{H}$, where $m_{H}$ is the mass of the scalar. Moreover, the photon becomes massive, with its Compton wavelength smaller than the size of the condensate ball [1]. We refer to these balls as metanuclei.

Although the easiest way to understand the (meta)stability of the metanuclei is in terms of the balance between the positive (outward) pressure of the bulk fermions, and negative pressure of the charged condensate, it is nevertheless useful to describe this in terms of the electrostatic interactions too. In the bulk of the metanucleus, there is a screened interaction between the negatively charged fermions and positively charged condensate. Furthermore, there is an attractive interaction between the charged condensate and surface fermions, and repulsion between the bulk and surface fermions, as well as between the surface fermions themselves.

In an analogous problem with positively and negatively charged classical particles in the bulk, and negatively charged particles on the surface of radius $R$, there would be an exact cancellation between the interactions of the positively and negatively charged bulk particles with the negatively charged surface particles; hence, such a surface would not be stable.

However, this is not the case in our problem. Charged condensate in the bulk differs from just a collection of classical charged particles. The former is a relativistic substance and its attractive interaction with the surface fermions scales differently

\footnotetext{
${ }^{1}$ We should also make sure that the fermions and scalars don't form neutral atoms, this could be arranged by increasing density and/or temperature of the system (see below).
} 
with $R$ 2. Moreover, in the regime when $N^{1 / 3} \ll g^{2} Q \ll g N^{2 / 3}$, the bulk-surface interactions, which end up being of order $N Q$, dominate over the net bulk-bulk (at most $\sim N^{4 / 3}$ ), and the surface-surface (at most $\sim Q^{2}$ ) interactions. Precisely it is in this regime that we find the local minimum of the energy functional.

In spite of a seeming mutual repulsion of the $Q$ fermions at the surface, it is energetically unfavorable for them to escape, as we will show in Section 4 . The presence of the $Q$ fermions on the surface, is the very reason for the existence of a constant gauge invariant potential in the bulk, which is responsible for keeping the bulk scalars in the condensate state. Hence, removal of surface fermions becomes energetically expedient, since it causes changes in the bulk energy.

In a certain respect, the metanuclei resemble properties of a metallic ball with an excess charg $\underbrace{3}$. In metals, the excess charge resides on the surface because it's energetically favorable to maintain zero electric field in the bulk. The surface charge, when it's not too large, in spite of its mutual repulsion, is not escaping the metallic ball (see more in Section 4).

The above construction seems generic. It could be applicable to systems that are described by a relativistic Abelian gauge theory. The scalars and fermions could be fundamental particles or composite states.

One application is to the system of $2 N+Q$ electrons, $e^{-}$, and $N$ helium- 4 nuclei, $\mathrm{He}^{++}$. We consider this system below the nuclear but above atomic densities, so that the nuclear effects are negligible, while the atoms are dissolved. We show that longlived charged metanuclei, made of the electrons and condensed $\mathrm{He}^{++}$states, may exist. These metanuclei are truly colossal - of the size of the Hydrogen atom or even greater - and carry enormous charge and excess energy. Such giant nuclei cannot form neutral atoms, making their survival in the Universe potentially difficult.

The metanuclei could also be "made of" other existing particles, such as pions, or still hypothetical particles, such as sleptons or squarks, if captured in the condensate before they decayed.

The organization of the paper is as follows: in Section 2 we zoom onto the interior of the condensate ball assuming that it fills entire space (this is a good approximation for the ball as long as its size is much greater than the width of its boundary region; we'll justify this assumption for the metanuclei in Section 3). We briefly summarize the results of [1] on condensation of charged scalars. In Section 3 we discuss the surface-bulk connection and show that the condensate ball is a (local) minimum of the energy functional. In Section 4 we discuss energetics of the charged condensate balls and study why a few possible decay channels of these balls can be

\footnotetext{
${ }^{2} \mathrm{~A}$ field they reason for this is that the expression for the current density of fermions does not depend on the gauge field, while that for a relativistic scalar manifestly depends on the gauge field via the covariant derivative. Note that in the Thomas-Fermi (or any other mean field) approach the effective fermion number density would depend on the value of the gauge potential, however, the latter would still be different from the dependence of the number density for the scalar.

${ }^{3}$ Except that, we do not expect the charged condensate to form a crystalline structure, since interactions in its bulk are screened, see discussions in [1].
} 
suppressed. In Section 5 we give some examples.

The metanuclei resemble a non-topological soliton (see, e.g., [2, 3, 4]) of a $Q$ ball type [2, 4, 5] with a local charge (charged Q-balls) [6] - especially the charged Q-balls with fermions [7]. However, there are important differences: the $Q$-balls require a special form of the non-linear potential for the scalar [2, 4, 6], while in our case the non-linear scalar self-potential plays no role - it is the scalar mass term and its interactions with the gauge field that are crucial. $Q$-balls could form due to a global minimum of the energy functional, while the metanuclei would form due to a local minimum.

\section{Dynamics in the interior (bulk) of the ball}

We start by considering a simple model of charged scalars and oppositely charged fermions at zero temperature. The classical Lagrangian contains a gauge field $A_{\mu}$, a charged scalar field $\phi$ with mass $m_{H}$, and fermions $\Psi^{+}, \Psi$ with mass $m_{J}$

$$
\mathcal{L}=-\frac{1}{4} F_{\mu \nu}^{2}+\left|D_{\mu} \phi\right|^{2}-m_{H}^{2} \phi^{*} \phi+\bar{\Psi}\left(i \gamma^{\mu} D_{\mu}-m_{J}\right) \Psi
$$

The covariant derivatives in (11) are defined as $\partial_{\mu}-i g A_{\mu}$ for the scalars and $\partial_{\mu}+i g A_{\mu}$ for the fermions. Although, for simplicity we have assumed that the scalar and fermion charges are equal, $g \equiv g_{\psi}=-g_{\phi}$, our results apply to a general cas 4 .

We introduce the following notations for the scalar, fermion, and gauge fields: $\phi=\frac{1}{\sqrt{2}} \sigma e^{i \alpha}, \Psi=\psi e^{-i \beta}, B_{\mu} \equiv A_{\mu}-\frac{1}{g} \partial_{\mu} \alpha$, and $\gamma \equiv \alpha-\beta$. In terms of the gauge invariant variables $\sigma, \psi, B_{\mu}$ and $\gamma$, the Lagrangian takes the form

$$
\mathcal{L}=-\frac{1}{4} F_{\mu \nu}^{2}+\frac{1}{2}\left(\partial_{\mu} \sigma\right)^{2}+\frac{1}{2} g^{2} B_{\mu}^{2} \sigma^{2}-\frac{1}{2} m_{H}^{2} \sigma^{2}+\bar{\psi}\left(i \gamma^{\mu} D_{\mu}-m_{J}\right) \psi-\left(\partial_{\mu} \gamma\right) \bar{\psi} \gamma^{\mu} \psi
$$

where now $F_{\mu \nu}$ and $D_{\mu}$ are the field-strength and covariant derivative for $B_{\mu}$, respectively. The key point for our discussions is that the third term in the Lagrangian (2) gives rise to a tachyonic mass for the scalar $\sigma$ if the field $g B_{0}$ acquires a vacuum expectation value [8, 9]. Moreover, when $\left\langle g B_{0}\right\rangle=m_{H}$, the scalar field condenses. Note that we retain the (last) total-derivative term in (2) as it will be important in our considerations.

To reach the condensate point, following Ref. [1], we assume that temperature is low-enough that the fermions form a degenerate system with overlapping de Broglie wavelengths, so that the fermions can be averaged over. Hence, we consider a system with a uniform background of fermions: $J_{\mu} \equiv \bar{\psi} \gamma_{\mu} \psi=J_{0} \delta_{\mu 0}$. The equations of motion derived from (2) are:

$$
-\partial^{\mu} F_{\mu \nu}=g^{2} B_{\nu} \sigma^{2}-g J_{\nu}, \quad \square \sigma=g^{2} B_{\mu}^{2} \sigma-m_{H}^{2} \sigma .
$$

\footnotetext{
${ }^{4}$ The conditions under which the other possible interactions in the Lagrangian (11) won't affect our conclusions were discussed in Ref. [1. For instance, there is a wide range of the parameter space where the possible scalar quartic coupling term is insignificant for our discussions.
} 
The theory admits a static solution with constant $B_{0}, \sigma$ :

$$
\left\langle g B_{0}\right\rangle=m_{H}, \quad\langle\sigma\rangle=\sqrt{\frac{J_{0}}{m_{H}}} .
$$

The quantity $-\left\langle g B_{0}+\dot{\gamma}\right\rangle$ acts as a dynamically induced chemical potential for the fermions, implying $\epsilon_{F}^{\prime}=-\left\langle g B_{0}+\dot{\gamma}\right\rangle$, where $\epsilon_{F}^{\prime}$ is the Fermi energy (unconventionally normalized, to include $B_{0}$ for simplicity). By this the negative value of the phase, $\dot{\gamma} \equiv \partial_{0} \gamma$, gets fixed.

For the scalars, it is the quantity $\left\langle g B_{0}\right\rangle=m_{H}$ that acts as an effective chemical potential 5 .

The bulk of the condensate is electrically neutral due to the compensation between the fermion and scalar charge densities: $g J_{0}-g^{2} B_{0} \sigma^{2}=0$. However, a nonzero $g B_{0}$ implies an uncompensated charge on a surface enclosing the condensate [1]. The spectrum of small perturbations above the condensate is composed of a scalar of mass $m_{s}=2 m_{H}$, and a photon that has acquired the mass $m_{g}=g \sqrt{J_{0} / m_{H}}$ (see, Ref. [1] for details).

The purpose of the present work is to show that such objects, with the surface charge and condensate bulk, can be long-lived. For notational simplicity, from now on we will be dropping the brackets, $\langle\cdot\rangle$, denoting the condensates.

\section{Surface-bulk connection}

We now consider a spherically symmetric system of a finite radius $R$ and look for a (meta)stable solution. We include the dynamics of the fermions in our considerations. The Hamiltonian derived from (2) is

$$
\begin{aligned}
\mathcal{H} & =\mathcal{H}_{\psi}+\frac{1}{4} F_{i j}^{2}+\frac{1}{2} \pi_{j}^{2}+B_{0}\left(\partial_{j} \pi_{j}+g J_{0}-\frac{1}{2} g^{2} B_{0} \sigma^{2}\right)+\frac{1}{2} P_{\sigma}^{2} \\
& +\frac{1}{2}\left(\partial_{j} \sigma\right)^{2}+\frac{1}{2} g^{2} B_{j}^{2} \sigma^{2}+\frac{1}{2} m_{H}^{2} \sigma^{2}
\end{aligned}
$$

where $\pi_{j} \equiv-F_{0 j}, P_{\sigma}=\dot{\sigma}$, are the canonical momenta for the $B_{j}$ and $\sigma$ fields respectively, and $\mathcal{H}_{\psi} \equiv i \bar{\psi} \gamma_{j}\left(\partial_{j}+i g B_{j}+i \partial_{j} \gamma\right) \psi+m_{J} \bar{\psi} \psi$, denotes the Hamiltonian density of the fermions. 6 .

\footnotetext{
${ }^{5}$ One could have also introduced a chemical potential $\mu_{s}$ for the scalars by adding the terms $+\mu_{s}\left(-g B_{0} \sigma^{2}\right)+\frac{1}{2} \mu_{s}^{2} \sigma^{2}$ to (2). However, we can absorb these terms into a redefinition of $B_{0}$ : $B_{0}^{\prime}=B_{0}-\frac{1}{g} \mu_{s}$.

${ }^{6}$ The quantity $\gamma$ is not a dynamical field. We will find a nonzero $\partial_{0} \gamma$ on the solution. The latter can be though of as the chemical potential for the fermions. Upon canonically transformation from the Lagrangian density (2) to the Hamiltonian density (5) the total derivative term with $\gamma$ disappears. As a result, the expression (5) corresponds to a Hamiltonian density $\mathcal{H}$, and not to the thermodynamic potential density, $\mathcal{H}^{\prime} \equiv \mathcal{H}-\mu J_{0}$, of the Grand Canonical partition function. The energy that is being minimized is determined by $\mathcal{H}$, while the equations of motion are those obtained from $\mathcal{H}^{\prime}$, i.e., one has to add to (5) the chemical potential term (expressed via $\dot{\gamma}$ ) to recover the correct Lagrangian equations of motion.
} 
As mentioned above, a nonzero $g B_{0}+\dot{\gamma}$ acts as an effective chemical potential for the fermions in the ball:

$$
\epsilon_{F}^{\prime} \equiv \sqrt{\left(3 \pi^{2} J_{0}\right)^{2 / 3}+m_{J}^{2}}=-\left(g B_{0}+\dot{\gamma}\right) .
$$

This defines the value of $\dot{\gamma}$ in the bulk to be $\dot{\gamma}=-\left(\epsilon_{F}^{\prime}+m_{H}\right)$, and we regard to $B_{0}$ as a part of the total chemical potential for the fermions 7 .

As $\dot{B}_{0}$ does not appear in the Lagrangian, the equation of motion for $B_{0}$ gives us Gauss's law:

$$
-\nabla^{2} B_{0}+\partial_{0} \partial_{j} B_{j}=g J_{0}-g^{2} B_{0} \sigma^{2} \equiv g J_{0}^{\text {total }} .
$$

Equation (7) has two important implications for the value of the fields in the bulk. The first is that in the bulk of the condensate where gradients are zero we have

$$
g B_{0}=\frac{J_{0}}{\sigma^{2}} .
$$

Secondly, equation (7) determines the value of $B_{0}$ in the bulk in terms of the conserved charge $Q$ and the radius $R$. Taking $\partial_{0} \partial_{j} B_{j}$ to be zero everywhere, we solve equation (7):

$$
B_{0}(r)= \begin{cases}\frac{g Q}{4 \pi R} & \text { for } r \leq R \\ \frac{g Q}{4 \pi r} & \text { for } r>R\end{cases}
$$

where $Q \equiv \int d^{3} r J_{0}^{\text {total }}$. We have set $B_{0} \rightarrow 0$ as $r \rightarrow \infty$, since $B_{0}$ is a gauge invariant variable, and a nonzero $B_{0}$ in the vacuum (i.e., far away from the condensate ball) would imply a different spectrum of the theory - a different mass for $\sigma$ and Lorentz violating interactions of $\sigma$ with the gauge field.

Therefore, we can use (77) to integrate out $B_{0}$ from the Hamiltonian, which becomes

$$
\mathcal{H}=\mathcal{H}_{\psi}+\frac{1}{2} \frac{J_{s}^{2}}{\sigma^{2}}+\frac{1}{2} m_{H}^{2} \sigma^{2}+\mathcal{H}_{\text {surface }}
$$

where $J_{s}=J_{0}+g^{-1} \partial_{j} \pi_{j}$ is the scalar charge density, and $\mathcal{H}_{\text {surface }}$ refers to all surface and gradient terms. In order not to select a preferred direction we set $B_{j}, J_{j}$ to zero.

Note that one can apply the scaling arguments to the expression for the energy functional that is obtained by integrating (10) w.r.t. $d^{3} x$. The scaling considerations

\footnotetext{
${ }^{7}$ One could apply the Thomas-Fermi (TF) approach to the system of fermions. In ordinary electrodynamics this would lead to a selfconsistent non-linear equation for the gauge potential. Here, the TF equation contains the phase of the charged scalar (or, equivalently, its gauge-invariant combination with the phase of the fermion, $\gamma$ ), which in our notations appears in the fermion equation as an effective chemical potential. The TF equation just determines that phase. The result for the fermions in the bulk of the ball is automatically accounted for by our eq. (6). We will use below the TF approach for the fermions near the surface, see eq. (14).
} 
give an opposite dependence on the scale parameter of the second and the third terms on the r.h.s. - one is a decreasing function of the scale parameter while the other one is an increasing function. This suggests that there should exist at least a local minimum of the energy functional due to the competition between these two terms, when they dominate over the others.

At this point we have used every equation of motion except for the equation of $\sigma$ and Gauss's law. For fixed scalar charge density $J_{s}$, the second and third terms on the r.h.s. of (10) could be thought of as an effective potential for the $\sigma$ field, in the regime where the field does not change significantly. In that regime, the above potential has a minimum. We vary (10) with respect to $\sigma$, ignoring all the gradient terms, and find, $\sigma=\left(J_{0} / m_{H}\right)^{1 / 2}$. Using this in equation (8) we find that $g B_{0}=m_{H}$. This is consistent with the solution of the previous section. Moreover, from (9) we deduce

$$
R_{c}=\frac{\alpha_{g} Q}{m_{H}}, \text { where } \alpha_{g} \equiv \frac{g^{2}}{4 \pi} .
$$

Thus, for a given $Q$, the radius of a ball of condensate is completely determined.

Furthermore, we wish to show that the radius (11) minimizes the energy of the condensate ball as a functions of $R$, in agreement with the scaling arguments. The formalism of the previous paragraph does not allow us to do so as $R$ is fixed on the solution. Instead we relax our enforcement of the equation for $\sigma$, and vary w.r.t. $R$. Our logic is as follows: In the bulk $B_{0}=g Q /(4 \pi R)$. In addition to the charge $Q$ being conserved, the total number of scalars $N_{s}$ is also conserved:

$$
N_{s}=\int d^{3} r g B_{0} \sigma^{2}
$$

Then, from the scaling of $B_{0}$ in the bulk, $B_{0}=g Q /(4 \pi R)$, it follows that $\int d^{3} r \sigma^{2} \sim$ $R$. Using these scalings in (10), the total energy dependence on $R$ can be read from:

$$
E=E_{\psi}+\frac{N}{2}\left(\frac{\alpha_{g} Q}{R}+\frac{m_{H}^{2} R}{\alpha_{g} Q}\right)+E_{\text {surface }}
$$

where $N \equiv \int d^{3} r J_{0}$ is the total number of fermions and $N \simeq N_{s}$ as long as $Q \ll N$. The first term on the r.h.s. of equation (13) is the energy of the free fermions which, due to their degeneracy pressure, tend to expand the ball of condensate. The first term in the parenthesis comes from the scalar-gauge and fermion-gauge field interaction terms and also provides positive pressure. The term $E_{\text {surface }}$ contains the non-relativistic part of the energy due to the surface charge, which works to expand the ball as well. It is only the second term in the parenthesis in (13), however, that provides the negative pressure and wants to contract the ball. Because this term contains $\sim \int d^{3} r \sigma^{2}$, it scales as $R$. We can use this negative pressure to stabilize the ball against the other terms. 
We chose to consider solutions where the repulsive term $\sim N Q / R$, and the attractive negative pressure term $\sim m_{H}^{2} N R / Q$ are dominant 8 . In the limit that the fermions are relativistic, this is true when $\alpha_{g} Q \gg N^{1 / 3}$. For non-relativistic fermions, the bound is $\alpha_{g} Q \gg\left(m_{H} / m_{J}\right)^{1 / 2} N^{1 / 3}$. In either case, the critical radius is in agreement with (11), obtained previously from the variation w.r.t. $\sigma$.

The exact static solution of the equations of motion (3) is hard to obtain. In Ref. [1] we found an approximate solution in the interior and exterior of the condensate ball. For generic values of the parameters, the obtained solutions are valid everywhere except in a very narrow region near the boundary of the ball, where our approximations break down 9 . Nevertheless, we matched the asymptotic solutions and their derivatives across the surface, demonstrating that with the asymptotic boundary conditions that we used, there are enough integration constants for the matching to be possible. The matching gave a relation between the critical radius $R_{c}$ and charge $g Q$ which closely approximates (11).

In our derivations of the solution in Ref. [1], and in its use here, we assumed that there is a bulk region in which the fermion number density is homogeneous, and that the size of this region is much greater than the surface width (i.e., we used the thin-wall approximation). Having made this assumption, we solved the coupled classical equations of motion and found that the width of the surface is determined by the scale $\left(m_{H} m_{g}\right)^{-1 / 2}$. The latter happens to be much smaller then the size of the bulk region, $R_{c} \simeq \alpha_{g} Q / m_{H}$, as long as $N Q \gg \alpha_{g}^{-1}$, which is the case here.

To complete the check of the thin-wall assumption the matching of the fermionic component should also be considered. For this, we use the Thomas-Fermi approach. Since the fermions couple to the net potential $B_{0}+\dot{\gamma}$, the TF equation in our case takes the form:

$$
-\nabla^{2} B_{0}+g^{2} B_{0} \sigma^{2}=\frac{g}{3 \pi^{2}}\left(\left(g B_{0}+\dot{\gamma}\right)^{2}-m_{J}^{2}\right)^{3 / 2} .
$$

The above equation can be solved inside and outside of the radius $R_{c}$. The interior solution coincides with that of the previous section. In the exterior, we obtain

$$
\dot{\gamma}_{\mathrm{ext}} \simeq-\frac{\alpha_{g} Q}{r}-\sqrt{\left(3 \pi^{2} g B_{0}^{\mathrm{ext}} \sigma_{\mathrm{ext}}^{2}\right)^{2 / 3}+m_{J}^{2}}
$$

where $B_{0}^{\text {ext }}$ and $\sigma_{\text {ext }}$ are the exterior solutions of [1]. The interior and exterior solutions for $\dot{\gamma}$ match at $r \simeq R_{c}$, as they should. This is enough for the complete matching, since in our formalism $\gamma$ has no second derivative term in the action. As an outcome of the above considerations, and using the fact that the $\sigma$ field vanishes

\footnotetext{
${ }^{8}$ We could also stabilize the negative pressure term against any other positive pressure terms in the above expression, e.g., against the fermion degeneracy pressure term. However, the solutions obtained by stabilizing against $E_{\psi}$ or $E_{\text {surface }}$ do not recover the infinite volume solution in the bulk of the ball. Although these solutions may well exist, their properties, such as a spectrum of small perturbations, would be different.

${ }^{9}$ The solutions are valid near the boundary as well only for particular values of the parameters.
} 
exponentially for $\left(r-R_{c}\right) \gg m_{H}^{-1}$, we find that $\dot{\gamma} \simeq-\left(\alpha_{g} Q / r\right)-m_{J}$, away from the ball.

As was pointed out above, the total effective potential for the fermions (the potential plus the chemical potential) is, $B_{0}+\dot{\gamma}$. Its value is a negative constant in the interior, $-\sqrt{\left(3 \pi^{2} J_{0}\right)^{2 / 3}+m_{J}^{2}}$, while in the exterior it asymptotes to $-m_{J}$. Therefore, the reletivistic fermions appear as if they're trapped in a potential well of the depth $\sim J_{0}^{1 / 3}$ and width $\sim \alpha_{g} Q / m_{H}$. This should certainly be so for an equilibrium state.

\section{Energetics}

In order to determine whether the condensate ball can be absolutely stable or not we should compare its energy with the total energy of $N$ neutral atoms formed by the scalars and fermions, and $Q$ free fermions. This energy is:

$$
E_{a}=\left(m_{H}-E_{b}\right) N+m_{J} Q
$$

where the binding energy is determined by $E_{b} \simeq\left(\alpha_{g}\right)^{2} m_{J} m_{H} / 2\left(m_{J}+m_{H}\right)$.

The energy of the condensate ball can be calculated from (13) using (11):

$$
E_{c}=E_{\psi}+m_{H} N+E_{\text {surface }} .
$$

The latter would always exceed (16). However, even when $E_{c}$ is greater than $E_{a}$ the condensate ball could be a long-lived as it represents a local minimum of the energy functional. In this case, it will be classically stable, however, would be able to decay through tunneling. To estimate the probability of the tunneling one could use an analog quantum mechanical decay rate,

$$
\Gamma \propto \exp \left(-\int_{R_{c}}^{R_{b}} d R\left(E(R)-E_{c}\right)\right),
$$

where $R_{b}$ is an initial radius of the ball after the tunneling. The ball could tunnel, while radiating away energy, directly into the size $R_{b} \sim R_{a} \equiv N^{1 / 3} /\left(\alpha_{g} m_{J}\right)$ that would allow the state of $N$ neutral atoms and $Q$ free charges to form. However, $R_{a}$ is much greater than $R_{c}$ according to our construction, and such a process would be highly suppressed. Instead, the ball could first tunnel into a state of a radius smaller than $R_{a}$ but greater that $R_{c}$, and then expand toward the state with neutral atoms. An estimate of the tunneling rate for the latter process could be obtained by assuming that $\left(R_{b}-R_{c}\right) \sim R_{c}$ and $\left(E\left(R_{b}\right)-E_{c}\right) \sim E_{c}$, this being justified when $m_{H}$ is the heaviest mass scale, and implies that individual particles have to overcome at least a potential barrier with the hight of order $\sim m_{H}$, and widths of order $\sim R_{c}$. Then, using the expressions $E_{c} \simeq m_{H} N$ and $R_{c}=\alpha_{g} Q / m_{H}$, we get the following scaling for the decay rate, $\Gamma \propto \exp \left(-k \alpha_{g} N Q\right)$, where $k$ is some undetermined numerical coefficient, which presumably is small at the scale set by 
$N$ and $Q$. Hence, for large values of $N$ and $Q$ the decay is strongly suppressed. Note that in this case the global and local vacua are not described by the same lowenergy degrees of freedom. The processes in which small regions of the true vacuum (i.e., the atomic phase, that necessarily has a lower particle number density) could materialize within the ball, would create local overdensities in the ball because of the particle number conservation, and would be exponentially suppressed at low temperatures.

The tunneling process discussed above describes the destruction of the whole metanucleus. The metanuclei may decay into their smaller counterparts via the tunneling of individual particles, or neutral pairs of particles escaping directly from the bulk of the nucleus. We can perform estimates similar to the one done above - to escape, a particle should at least overcome a potential barrier with the hight of order $\sim m_{H}$, and widths of order $\sim R_{c}$. Then, the tunneling rate would be suppressed at least by the exponential factor, $\exp \left(-\alpha_{g} Q\right)$ (we ignore the numerical coefficient in the exponent). This is strongly suppressed for large values of $Q$.

There are other channels through which a ball of condensate could decay. We start with the decay through the evaporation of surface charges or, similarly, the accretion of nearby charges, if the latter are present. On the solution the ratio $Q / R$ is fixed, $Q / R=m_{H} / \alpha_{g}$. A spontaneous emission of a single charge from the surface would result in a new radius $R^{\prime}=(Q-1) R / Q$, with reduced surface energy. However, this would lead to the growth of the bulk Fermi degeneracy energy. To study systematically whether the emission process is favorable or not, we fix the ratio $Q / R$ and vary the energy with respect to $R$. Including the energy of the fermions and of the surface charge, the total energy (in the relativistic approximation for the fermions) is

$$
E=\frac{3}{4}\left(\frac{9 \pi}{4}\right)^{1 / 3} \frac{N^{4 / 3}}{R}+\frac{m_{H}^{2}}{\alpha_{g}} R+m_{H} N .
$$

We have ignored the gradient of $\sigma$ in the bulk and near the surface, which in any event are $\lesssim N / R$, and, hence negligible.

Varying with respect to $R$ gives $R_{\text {optimal }} \propto \alpha_{g}^{1 / 2} N^{2 / 3} / m_{H}$. Since emitting a charge decreases $R$, we want $R_{c}<R_{\text {optimal }}$ in order for the condensate ball to be stable with respect to emission. This implies that $\alpha_{g} Q \ll \alpha_{g}^{1 / 2} N^{2 / 3}$. Thus, combining all the constraints, our solution is valid as long as

$$
1 \ll N^{1 / 3} \ll \alpha_{g} Q \ll \alpha_{g}^{1 / 2} N^{2 / 3}
$$

For $Q>\alpha_{g}^{1 / 2} N^{2 / 3}$ the condensate ball will emit charges, or decay into smaller balls (fission of metanuclei), until $R_{c}=R_{\text {optimal }}$. Furthermore, when (20) is satisfied, and there are other charges present nearby, it is possible for the condensate ball to accrete charges, or to fuse with other balls, until the stable radius is reached 10 .

${ }^{10}$ For the nonrelativistic fermions the condition of stability $(20)$ becomes $Q \lesssim\left(\frac{m_{H}}{m_{J}}\right)^{1 / 3} N^{5 / 9}$. 
The above energetics arguments show that the electrons would not escape the surface, as long as (20) is satisfied. There should also exist a microscopic explanation for this, in terms of the local attractive forces acting on the surface electrons. Perhaps, the most straightforward explanation would have been in terms of (quasi)localization of the fermions due to the bosonic background near the surface. This is plausible, since the scaling of the attractive interactions between the bulk condensate and the surface fermions, differs from that of the repulsion between the bulk and surface fermions. However, a rigorous study of this issue would require exact solutions for the scalar and gauge fields within the surface layer, which are not available at present. There are also different mechanisms that may also contribute to an effective attraction for the surface electrons.

The first mechanism is based on an analogy with an excess charge on the surface of a conductor. As it is known, up to a certain critical value, this excess charge would not escape the surface. In terms of energetics, this can be explained as follows: if a single negative charge is being slightly removed from the surface, a positive imagecharge should be put in the place of the removed negative one, in order to maintain the equipotential surface [10]. In terms of local interactions, this can be understood in terms of the so-called double layer (dipole-like) structure that appears near the surface of a conductor [11]. Let us first consider a conductor with zero overall charge. Because the electrons in the conductor are bound weaker than the lattice ions, there is a slight leakage of the electronic charge beyond the surface, when the latter is defined according to the distribution of the lattice ions. Let us denote the extent of the leakage by $L$, measured as a distance from the surface. Hence, at a distance $L$ in the exterior of the surface there is an excess of negative charges $N_{-}$, and therefore, at a distance $L$ in the interior, there is an excess of positive charges $N_{+}$. Then, according to Gauss's law, any negative charge placed outside of the surface at a distance less than $L$, would experience a local attraction toward the surface. This is what's called the double layer attraction for the neutral conductors. The above mechanism remains approximately valid even when additional charge $g Q$ is placed on the surface, as long as $g Q \ll N_{+}$.

Similar arguments could be presented for the surface physics of metanuclei. The scalars and fermions, spill out of the surface just a bit, to a small scale of order $\Delta \sim\left(m_{H} m_{g}\right)^{-1 / 2}$. It's only within this scale that the dipole layer may form because of the difference between the scalar and fermion masses and interactions. If so, we can estimate the dipole charge to be $N_{+} \sim g J_{s} R_{c}^{2} \Delta$. Then, the double-layer attraction will be present, as long as the charge $N_{+}$is much greater that the excess charge $g Q$. The latter condition gives $\alpha_{g} Q \ll \alpha_{g}^{1 / 5} N^{3 / 5}$. Typically, this would a bit stronger than the constraint in (20).

As to the second possible mechanism, there may be a local attraction of like charges near the surface of the ball due to relativistic effects. Consider a single electron near the surface of a negatively charged ball that we're dealing with. It's been long known (see, e.g., [13, 14]) that once the magnitude of the electrostatic potential exceeds the electron mass, relativistic attraction effects, determined by 
minus the potential square, may dominate over the nonrelativistic repulsion (we note here that this attraction, however, cannot lead to quantum-mechanical bound states for Dirac equation). The electrons near the surface in our case are in this relativistic regime; for instance, in one of the example considered in the next section the total relevant potential for surface electrons (that also includes their chemical potential) is $\sim 5 \mathrm{MeV}$, which is about 10 times greater than the electron mass.

Whether the above two mechanisms make significant contributions to the nearthe-surface attraction, remains to be seen.

Another potential decay channel is via Schwinger pair-creation of the fermions (we assume that the fermions are lighter than the scalars in our case) or other light charged particles, due to the electric field near the surface of the ball. We consider first large size metanuclei (the ones that because of their size cannot have deep bound levels [14]), for which the electric field $\mathcal{E}=\frac{g Q}{4 \pi R^{2}}=\frac{4 \pi m_{H}^{2}}{g^{3} Q}$ can be made subcritical by increasing $Q$. The standard textbook formula for the pair-creation in a constant electric field may not be applicable here, since the process involves tunneling to infinity, in which case the metanuclei, no matter how large, cannot be well-approximated by an infinite charged plane. Nevertheless, one can estimate the pair-creation probability of particles of mass $m<m_{H}$ by the quasi-classical exponent, $\mathcal{W} \propto \exp (-2 S)$

$$
S=\int_{R_{0}}^{\infty}|p(R)| d R, \quad|p(R)| \equiv \sqrt{\left(2 m \alpha_{g} Q / R\right)-\left(\alpha_{g}^{2} Q^{2} / R^{2}\right)}
$$

where $R_{0} \equiv \alpha_{g} Q / 2 m$. When the upper limit in the integral above is set to infinity, corresponding to creation of a on-shell pair, the integral diverges, and the probability is zero. This is consistent with our earlier finding that the electrons do not escape from the surface, because of the energetics arguments. In more general cases, however, particles can be created with a nonzero energy, which can happen when one of them ends up in a state of a negative energy binding with the metanucleus, ensuring by its binding the conservation of energy 11 .

In this case, (21) would get modified. Most significantly, the upper limit of the integration in (21) would become a finite, energy dependent, number. However, the exponential factor would still be proportional to $S \propto \alpha_{g} Q$, and the probability would be suppressed for large values of $Q$. This is in accordance with the intuition that the electric field of the metanucleus decreases with increasing $Q$. The exponent giving the dominant contribution, when the high energy particle production is allowed by the energy conservation, would scale as $S \sim \alpha_{g} Q\left(m^{2} / m_{H}^{2}\right) \sim\left(m^{2} / g \mathcal{E}\right)$, in a qualitative agreement with the Schwinger formula.

In the next section we consider the metanuclei made of the helium- 4 ions and electrons at densities above atomic and below nuclear. These metanuclei are of

\footnotetext{
${ }^{11}$ Alternatively, a positive energy could also be gained if a particle of the same charge as the metanucleus, attached itself to the metanucleus and increased its charge and size. However, in this case, since the partner particle is oppositely charged, the electric field of the metanucleus would not push it out to infinity to separate it from its pair.
} 
super-atomic size, and satisfy the above-discussed conditions of stability. On the other hand, one could also imagine metanuclei made of other scalars, such as e.g. sleptons. In this case the metanuclei can have a typical size of the ordinary nuclei. Then, the deep bound levels would be allowed, and the pair creation process won't be suppressed (for a review, see, [14]). The resulting equilibrium object would have a shell of induced screening charge around it. The calculation of the distribution of the screening charge for that case will be presented elsewhere.

\section{$5 \quad$ Metanuclei from electrons and helium nuclei}

The results of the previous sections can be adopted to the system of charged helium- 4 nuclei $\mathrm{He}^{++}$, and electrons $e^{-}$(the scalar charge $g_{\phi}$ is twice as large as the fermion charge $g_{\psi}$ ). We consider such a system below the nuclear but above the atomic density. The former condition sets $Q \gg \frac{m_{H}}{200 \mathrm{MeV}} \frac{N^{1 / 3}}{\alpha_{\mathrm{em}}}$, and the latter gives $Q \ll$ $\frac{1}{\alpha_{\mathrm{em}}^{2}} \frac{m_{H}}{m_{J}} N^{1 / 3}$.

Taking $m_{H} \simeq 3.7 \mathrm{GeV}, m_{J} \simeq 0.5 \mathrm{MeV}$, we find that the system with $N \sim$ $\left(10^{12}-10^{15}\right)$ and $Q \sim\left(10^{8}-10^{9}\right)$ satisfies all the constraints discussed in the previous sections 12 . The size of the condensate ball in this case is $R_{c} \sim\left(10^{5}-10^{6}\right) \mathrm{fm}$, with the average inter-particle separation $\sim(10-100) \mathrm{fm}$, the number-density of particles $\sim(2-20 \mathrm{MeV})^{3}$, and the total energy $E_{c} \sim 4 \cdot\left(10^{12}-10^{15}\right) \mathrm{GeV}$. These object have a huge energy excess - almost $40 \mathrm{MeV}$ per $\mathrm{He}^{++}$particle, in the simplest case. The excess energy per particle scales as $\sim m_{H} \frac{N^{1 / 3}}{\alpha_{\mathrm{em} Q}}$. There'll be huge energy liberated in decays of such metanuclei.

As long as temperature of the interior of the metanuclei is small enough that the $\mathrm{He}^{++}$de Broglie wavelengths still overlap, the above described properties are expected to remain valid 13 . For instance, for the number density $\sim(10 \mathrm{MeV})^{3}$, at temperatures below $10^{-2} \mathrm{MeV} \sim 10^{8} \mathrm{~K}$, the above described properties should be expected to hold. The metanuclei may have formed in starts, galaxies, or during some dramatic astrophysical events. They could represent a new state of matter, which could be searched for in, e.g., cosmic rays. One should expect, though, that their formation and survival probability in the Universe to be rather low. Identification of the concrete mechanisms of their formation in a cosmological/astrophysical environment, if such mechanism exist, requires further careful studies of finite temperature effects, and goes beyond the scope of the present work.

Similar condensate balls can be "made of" other scalars and fermions. Some examples are: (i) The scalars are $\mathrm{He}^{++}$nuclei and fermions are anti-protons; (ii) The scalars are condensed composite states such as Cooper pairs, or charged pions $\pi^{ \pm}$and fermions are anti-protons/protons or electrons/positrons; (iii) In supersymmetric

\footnotetext{
${ }^{12}$ There are other allowed possibilities for $N$ and $Q$. We choose the above numbers as typical.

${ }^{13}$ For low enough temperatures and densities, the fusion of the condensed helium nuclei, as well as the process of their destruction by energetic electrons, are expected to be suppressed. For instance, we consider densities that are below the "neutronization" threshold of the helium nuclei.
} 
models the role of the scalars could be played by squarks or sleptons. The helium-4 nuclei have an advantage that they are stable states. All the particles that can decay, such as the pions, squarks and sleptons, should be captured/produced in the condensate before they could decay.

The survival probability of some of the metanuclei would increase if they could form neutral meta-atoms by dressing up with electron/positrons. This possibility is planned to be discussed in future.

Acknowledgments. We'd like to thank Savas Dimopoulos, Lance Dixon, Misha Shifman, Matt Kleban, and especially, Andrei Gruzinov for valuable comments. The work of GG is supported by NASA grant NNGG05GH34G. RAR is supported by James Arthur graduate fellowship.

\section{References}

[1] G. Gabadadze and R. A. Rosen, Phys Lett. B 658 (2008), 266.

[2] G. Rosen J. Math. Phys. 9 (1968) 996.

[3] R. Friedberg, T. D. Lee and A. Sirlin, Nucl. Phys. B 115, 1 (1976).

[4] S. R. Coleman, Nucl. Phys. B 262, 263 (1985) [Err-ibid. B 269, 744 (1986)].

[5] A. Kusenko, Phys. Lett. B 404, 285 (1997) arXiv:hep-th/9704073].

[6] K. M. Lee, J. A. Stein-Schabes, R. Watkins and L. M. Widrow, Phys. Rev. D 39, 1665 (1989).

[7] K. N. Anagnostopoulos, M. Axenides, E. G. Floratos and N. Tetradis, Phys. Rev. D 64, 125006 (2001) arXiv:hep-ph/0109080].

[8] A. D. Linde, Phys. Rev. D 14, 3345 (1976).

[9] J. I. Kapusta, Phys. Rev. D 24 (1981) 426.

[10] J.D. Jackson, "Classical Electrodynamics", Wiley (1975).

[11] J. Frenkel, Z. Physik 51, 232 (1928).

[12] J. Bardeen, Phys. Rev. 49, 653 (1936).

[13] K. M. Case, Phys. Rev. 80, 797 (1950).

[14] Ya. B. Zel'dovich, V.S. Popov, Usp. Fiz. Nauk, 105 (1971) 403; [Sov. Phys. Usp., 14 (1972) 673]. 